\title{
DESAFIOS PARA EL PERU: APEC Y TLG
}

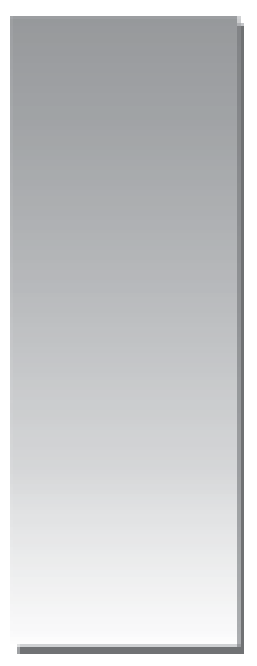

Pablo Mauricio Pachas (*)

E-mail:pwmauricio@yahoo.es

\begin{abstract}
RESUMEN
Son pocos los que conocen la importancia del Foro de Cooperación Económica del Asia Pacífico (APEC); ya que más se ha discutido sobre el Tratado de Libre Comercio (TLC) con Estados Unidos. En el presente artículo se señala la importancia de Asia en la economía mundial; se realiza una breve caracterización de los principales mercados asiáticos (China, Japón, Corea del Sur y Taiwán), y se analizan las posibilidades del Perú de constituirse en una plataforma estratégica para el comercio sudamericano con Asia, en el marco de la APEC. Asimismo, se discute las expectativas de abrir nuevos mercados con los principales bloques comerciales a través de Tratados de Libre Comercio.
\end{abstract}

Palabras clave: Foro de Cooperación Económica del Asia-Pacífico (APEC), Comercio Exterior PerúAsia:, Tratado de Libre Comercio.

\begin{abstract}
About APEC few people know its importance, but it has been discussed about Tried Free Commerce (TLC) with United States. In this article the importance of Asia in the world economy is indicated, a brief characterization of the main Asian markets is carried out (Chinese, Japan, South Korea and Taiwan), and is analyzed the possibilities of Peru to be constituted in a strategic platform for the South American commerce with the Asia in the framework of the APEC. Also, it is discussed the expectations to open new markets with the main commercial blocks through Tried Free Commerce.
\end{abstract}

Key words: Forum of Economic Cooperation of the Asia-Pacific, Foreign trade Peru-Asia , Free Trade Agreement

\section{INIRODUCCÓN}

Por su ubicación geográfica, nuestro país es considerado como plataforma estratégica para el comercio con los países del Asia, y en el 2008 será sede de la Reunión Mundial de Presidentes de los veintiún países que conforman el Foro de Coope- ración Económica del Asia Pacífico (APEC), año en que el Perú ejercerá la Presidencia de tan importante Foro.

Pocos conocen la importancia de APEC, porque más se ha discutido sobre el Tratado de Libre Comercio (TLC) con Estados Unidos, debi

(*) Magíster en Economía. Economista. Pasantía en la Bolsa de Valores de Nueva York (New York Stock Exchange - NYSE), Estados Unidos. Actualmente Consultor en Negocios Bursátiles; Profesor Principal y Director de la Escuela de Negocios Internacionales de la Facultad de Ciencias Administrativas (UNMSM). 
tradicionales predominantemente textiles y agroindustriales, en el marco de los beneficios que ha generado el ATPDEA próximo a terminar, preocupaciones que trataré de abordar en el presente articulo. Se destacará la importancia de los mercados de los países asiáticos así como las posibilidades que tiene el Perú para constituirse en la plataforma estratégica del comercio sudamericano con Asia, en el marco de APEC. Asimismo, trataré la importancia del TLC, previo repaso sobre los diferentes bloques comerciales existentes y las expectativas de abrir nuevos mercados con la Unión Europea y otras economías a través de TLCs.

\section{IMPORTANCIA DE ASIA EN LA ECONOMÍA MUNDIAL}

Asia cuenta con tres de la diez economías más grandes (China, Japón e India), y concentra a más del $35 \%$ de la producción mundial. Actualmente es la región con mayor y más rápido crecimiento del mundo, debido a los grandes esfuerzos realizados para integrarse a la economía mundial, y ahora capta alrededor del $40 \%$ del capital privado neto que fluye a los mercados emergentes. Según algunos análisis en los próximos años representará más del $50 \%$ de la economía mundial, lo que se explica -en gran medida- por los crecientes resultados que vienen obteniendo India y China.

\section{PRINCIPALES MERCADOS ASIÁTICOS}

\section{China}

La economía de China, durante el ultimo cuarto de siglo, ha pasado de un sistema centralista y cerrado a una economía abierta al comercio internacional, con un sector privado que crece rápidamente y es cada vez mayor, convirtiéndose así en uno de los países mas importantes en la economía global.

Las reformas comenzaron a finales de los 70 , con la eliminación progresiva de la agricultura colectivizada; luego incluyeron la liberalización gradual de precios, la descentralización fiscal, la autonomía creciente para las empresas de estado, creación de un sistema de actividades bancarias diversificadas, el desarrollo de los mercados de acciones, el crecimiento rápido del sector privado, y la apertura al comercio exterior y a la inversión.

El proceso continúa: en el 2005 se vendieron acciones de los bancos estatales más grandes de China a inversionistas extranjeros; se reestructuró la economía y se incrementó la eficiencia, lo que ha permitido que el PBI de China se multiplique por más de diez veces desde 1978. Medido sobre la base de la paridad de poder adquisitivo (PPP), China en el 2005 aparece como la segunda economía más grande en el mundo después de los Estados Unidos, aunque en términos per cápita, sigue siendo un país de

Gráfico 1

Flujo comercial del Perú con China (1997 - 2005)

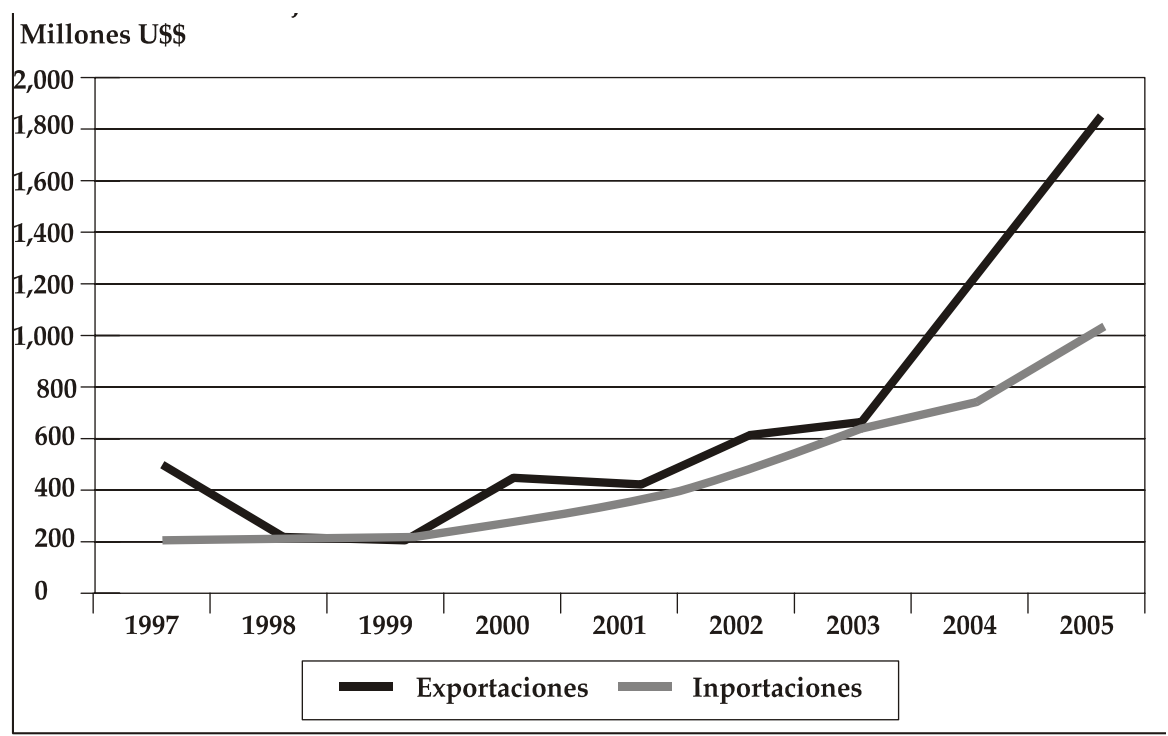


ingreso medio bajo, con 150 millones de chinos que caen debajo de las líneas internacionales de pobreza.

Su desarrollo económico ha sido generalmente más rápido en provincias costeras que en el interior, y existen grandes disparidades en la renta per cápita entre las regiones.

La importancia de China se puede resumir en las siguientes cifras: en el 2006 su población alcanzó los 1,313’973,713 habitantes; en los últimos 25 años ha venido creciendo a un ritmo de 9.9\% del PBI (2005), del orden de US\$ 2,225 trillones (2005) y con un PBI per cápita de US\$ 6,800, también en el 2005.

\section{Japón}

La cooperación entre el Gobierno y la industria, la ética del trabajo fuerte, la maestría de la alta tecnología, y la baja asignación de recursos a la defensa (el 1\% del GDP), ayudaron a Japón a crecer con una rapidez extraordinaria, convirtiéndose en la segunda economía tecnológica en el mundo después de los Estados Unidos, y en la tercera economía mas grande del mundo después de Estados Unidos y China, dato que resulta sobre la base de la paridad de poder adquisitivo (PPP).

Una notable característica de su economía es la manera como fabricantes, surtidores, y distribuidores trabajan de manera cercana y unida a través de grupos llamados keiretsu. Una segunda característica básica ha sido la garantía del empleo de por vida para una porción impor- tante de la mano de obra urbana. Ambas características están ahora desapareciendo.

El sector industrial de Japón es grandemente dependiente de materias primas y combustibles importados. El pequeño sector agrícola se subvenciona y se protege al punto de ser considerado uno de los más protegidos del mundo, aunque es autosuficiente en la producción de arroz. Sin embargo Japón debe importar cerca del $60 \%$ de sus alimentos, mantiene una de las flotas pesqueras más grandes del mundo y pesca casi el $15 \%$ de la pesca mundial.

Por tres décadas, el desarrollo económico de Japón había sido espectacular, con un promedio de $10 \%$ en los años $60,5 \%$ en los años 70 , y $4 \%$ en los años 80. Sin embargo, en los años 90 el crecimiento se contrajo, alcanzando un promedio de apenas $1.7 \%$, debido en parte a los efectos de la inversión excesiva a finales de los 80 y a las políticas contractivas del gobierno, orientadas a evitar los flujos especulativos de los mercados bursátiles y de las propiedades inmobiliarias, además de forzar a una reestructuración de la economía.

Del 2000 al 2003, los esfuerzos del gobierno se han orientado a restablecer el desarrollo económico, pero tuvieron poco éxito y fueron obstaculizados por el enfriamiento de la economía de Estados Unidos y la Unión Europea y el crecimiento de las economías asiáticas. En el 2004 y el 2005 el crecimiento mejoró, y el miedo persistente a la deflación de precios condujeron a que la actividad económica disminuyera.

Cuadro 1

Ranking de Economías

\begin{tabular}{|r|c|c|c|c|c|}
\hline $\mathbf{1}$ & EE.UU. & EE.UU. & EE.UU. & EE.UU. & EE.UU. \\
\hline $\mathbf{2}$ & JAPÓN & JAPÓN & JAPÓN & CHINA & CHINA \\
\hline $\mathbf{3}$ & ALEMANIA & ALEMANIA & CHINA & JAPÓN & JAPÓN \\
\hline $\mathbf{4}$ & $\begin{array}{l}\text { REINO } \\
\text { UNIDO }\end{array}$ & CHINA & ALEMANIA & ALEMANIA & ALEMANIA \\
\hline $\mathbf{5}$ & CHINA & $\begin{array}{l}\text { REINO } \\
\text { UNIDO }\end{array}$ & $\begin{array}{l}\text { REINO } \\
\text { UNIDO }\end{array}$ & $\begin{array}{l}\text { REINO } \\
\text { UNIDO }\end{array}$ & $\begin{array}{l}\text { REINO } \\
\text { UNIDO }\end{array}$ \\
\hline $\mathbf{6}$ & FRANCIA & FRANCIA & FRANCIA & FRANCIA & INDIA \\
\hline 7 & ITALIA & ITALIA & ITALIA & INDIA & FRANCIA \\
\hline $\mathbf{8}$ & INDIA & INDIA & INDIA & ITALIA & ITALIA \\
\hline
\end{tabular}


La enorme deuda que tiene el gobierno Japonés y que alcanza el $170 \%$ del GDP, aunada al envejecimiento de su población, son dos problemas duraderos que hay que tener en cuenta. La importancia de Japón se puede resumir en las siguientes cifras: en el 2006 su población llegó a 127’463,611 habitantes (2006), el PBI crece a un ritmo de $2.7 \%$ anual (2005), lo que determina un PBI de US\$ 4.664 trillones (2005) y un PBI per cápita de US\$ 31,500 (2005).

\section{Corea del Sur}

Desde inicios de los 60 este país ha alcanzado un increíble crecimiento económico, además de integrarse con la economía moderna de la alta tecnología. Hace cuatro décadas, el PBI per cápita era comparable con los países más pobres de África y Asia.

En el 2004 Corea del Sur ingresó al club de las economías mundiales que superan el trillón de dólares, y hoy en día su PBI per cápita es igual al de las pequeñas economías de la Unión Europea. Este éxito fue alcanzado a finales de los 80 gracias a un sistema de estrechos lazos entre el gobierno y los negocios privados, que incluyó crédito dirigido, restricciones de importación, patrocinio de industrias específicas, y trabajo fuerte. El gobierno promovió la importación de materias primas y de tecnología a expensas de bienes de consumo, además de motivar los ahorros e inversiones en vez del consumo.

La crisis financiera asiática de 1997-99 expuso debilidades acumuladas durante muchos años en el modelo de desarrollo de Corea del Sur, incluyendo altos cocientes de deuda/patrimonio, préstamo extranjero masivo, y un sector financiero indisciplinado. El PBI decreció en 6.9\% en 1998, pero tuvo una rápida recuperación alcanzando un crecimiento de $9.5 \%$ en 1999 y de $8.5 \%$ en el 2000. El crecimiento bajó de nuevo a $3.3 \%$ en el 2001, debido a que la economía global se estancó, las exportaciones cayeron y las reformas corporativas y financieras, muy necesarias, se entramparon. Impulsado por el gasto en el consumo y por las exportaciones, el crecimiento en el 2002 creció a 7\%, a pesar de un bajo crecimiento global.

Entre el 2003 y 2005 el crecimiento se moderó en cerca de $4 \%$. Un descenso en el gasto del consumidor fue compensado por un rápido crecimiento en la exportación. En el 2005 el gobierno propuso la reforma de la legislación laboral; un esquema de pensión corporativa para que el mercado de trabajo sea más flexible, y nuevas políticas para evitar la especulación sobre las propiedades inmobiliarias.

Hoy en día el país goza de una inflación moderada, bajo índice de desempleo, superávit comercial y una distribución muy parecida de la renta, características resaltantes de esta sólida economía. La importancia de Corea del Sur se puede resumir en las siguientes cifras: su población alcanza los 48'846,823 habitantes (2006); tiene un crecimiento de $3.9 \%$ anual del PBI (2005), que alcanza los US\$ 965.3 billones (2005), y su PBI per cápita asciende a US\$ 20,400 (2005 estimado).

\section{Taiwán}

Tiene una dinámica economía capitalista, donde las autoridades del gobierno disminuyen gradualmente el control sobre la dirección que deben de seguir las inversiones y el comercio exterior. En armonía con esta tendencia, se están privatizando algunos bancos y grandes firmas industriales que pertenecen al Estado.

Las exportaciones han sido el motor de la industrialización de Taiwán, contando con un elevado superávit comercial y reservas en el extranjero considerada como la tercera más grande del mundo. Ello motivó algunos cambios: por ejemplo, en 1952 la agricultura contribuía con $32 \%$ del PBI, y hoy en día aporta menos del $2 \%$.

La isla es un inversionista importante a través del Asia sur oriental. Actualmente China y Estados Unidos se han convertido en los mercados más grandes para las exportaciones de Taiwán. En el 2005 China Continental fue considerada como la tercera fuerza proveedora de importaciones de Taiwán, después de Japón y Estados Unidos.

Taiwán se ha beneficiado de la integración económica con China y de un claro aumento en la demanda mundial, lo que le ha permitido alcanzar un crecimiento importante en su sector exportador. Además, durante 7 años ha tenido un crecimiento económico sostenido, de $6.1 \%$ del PBI en 2004. Sin embargo, un exceso de inventario, los altos precios del petróleo y el cre- 


\section{Cuadro 2}

Exportaciones del Perú a los principales Mercados Asiáticos (1998-2002)

(En miles de dólares)

\begin{tabular}{|l|r|r|r|r|r|r|}
\hline \multicolumn{1}{|c|}{ Socio } & \multicolumn{1}{c|}{$1 \mathbf{1 9 9 8}$} & \multicolumn{1}{c|}{$\mathbf{1 9 9 9}$} & \multicolumn{1}{c|}{$\mathbf{2 0 0 0}$} & \multicolumn{1}{c|}{$\mathbf{2 0 0 1}$} & \multicolumn{1}{c|}{$\mathbf{2 0 0 2}$} & \multicolumn{1}{c|}{ Valor de 5 } \\
\hline Mundial & $5,671,812$ & $5,932,062$ & $6,866,047$ & $6,825,611$ & 490,425 & $32,785,956$ \\
\hline China & 233,179 & 215,496 & 442,678 & 426,253 & 596,891 & $1,914,497$ \\
\hline Japón & 216,350 & 257,728 & 325,387 & 383,017 & 372,608 & $1,555,090$ \\
\hline Corea del Sur & 41,950 & 60,998 & 137,589 & 110,592 & 168,082 & 519,211 \\
\hline Taiwán & 104,170 & 112,621 & 96,541 & 85,889 & 110283 & 509,504 \\
\hline Tailandia & 24,861 & 48,580 & 74,866 & 70,293 & 25,880 & 244,480 \\
\hline India & 24,270 & 46,816 & 34,756 & 36,460 & 21,142 & 163,444 \\
\hline Indonesia & 10,129 & 21,632 & 34,403 & 39,236 & 24,841 & 130,241 \\
\hline Hong Kong & 41,090 & 12,801 & 21,148 & 21,269 & 31,974 & 128,282 \\
\hline Filipinas & 2,162 & 29,309 & 38,996 & 20,436 & 13,052 & 123,960 \\
\hline Malasia & 20,021 & 20,108 & 22,225 & 14,767 & 9,340 & 86,461 \\
\hline Singapur & 7,538 & 4,388 & 3,584 & 7,916 & 6,713 & 30,139 \\
\hline Vietnam & 314 & 2,876 & 4,170 & 8,666 & 4,556 & 20,592 \\
\hline Total Asia & $\mathbf{7 4 6 , 0 3 9}$ & $\mathbf{8 3 3 , 3 5 3}$ & $\mathbf{1 , 2 3 6 , 3 4 3}$ & $\mathbf{1 , 2 2 4 , 7 9 4}$ & $\mathbf{1 , 3 8 5 , 3 7 2}$ & $\mathbf{5 , 4 2 5 , 9 0 1}$ \\
\hline
\end{tabular}

cimiento de las tasas de interés, menguaron el consumo en los mercados desarrollados; por lo cual, el crecimiento del PBI ha caído a 3.8\% en el 2005. El sector de servicios, que representa el 69\% del PBI de Taiwán, ha continuado ampliándose, mientras que las tasas del desempleo y de inflación han declinado.
La importancia de Taiwán se puede resumir en las siguientes cifras: su población alcanza los 23'036,087 habitantes (2006), su PBI tiene un crecimiento anual del 3.8\% (2005), y actualmente asciende a US\$ 631.2 billones (2005) y el PBI per cápita llega a US\$ 27,600 (2005 estimado).

Gráfico 2

\begin{tabular}{|l|r|r|r|r|r|r|}
\hline \multicolumn{1}{|c|}{ Socio } & 1998 & 1999 & 2000 & 2001 & \multicolumn{1}{c|}{2002} & Valor de 5 \\
\hline MUNDIAL & 5671812 & 5932062 & 6866047 & 6825611 & 7490425 & 32785956 \\
\hline CHINA & 233179 & 215496 & 442678 & 426253 & 596891 & 1914497 \\
\hline JAPAN & 216350 & 257728 & 325387 & 383017 & 372608 & 1555090 \\
\hline KOREA REP. & 41950 & 60998 & 137589 & 110592 & 168082 & 519211 \\
\hline TAIWAN (POC) & 104170 & 112621 & 96541 & 85889 & 110283 & 509504 \\
\hline THAILAND & 24861 & 48580 & 74866 & 70293 & 25880 & 244480 \\
\hline INDIA & 24270 & 46816 & 34756 & 36460 & 21142 & 163444 \\
\hline INDONESIA & 10129 & 21632 & 34403 & 39236 & 24841 & 130241 \\
\hline HONG KONG & 41090 & 12801 & 21148 & 21269 & 31974 & 128282 \\
\hline PHILIPPINES & 2162 & 29309 & 38996 & 20436 & 13052 & 123960 \\
\hline MALAYSIA & 20021 & 20108 & 22225 & 14767 & 9340 & 86461 \\
\hline SINGAPORE & 7538 & 4388 & 3584 & 7916 & 6713 & 30139 \\
\hline VIET NAM & 314 & 2876 & 4170 & 8666 & 4566 & 20592 \\
\hline Total Asia & 746039 & 833353 & 1236343 & 1224794 & 1385372 & 5425901 \\
\hline
\end{tabular}




\section{LAS RELACIONES COMERCIALES PERÚ - ASIA}

Durante los últimos años, el Perú ha realizado esfuerzos para ampliar el número de socios comerciales. En el Cuadro 2 presentamos los principales destinos Asiáticos de las exportaciones peruanas (China, Japón, Corea, Taiwán, Tailandia, India, Indonesia, Hong Kong, Filipinas, Malasia, Singapur y Vietnam). Respecto a inversiones peruanas en el Asia, cabe destacar la reciente planta industrial de Kola Real (empresa de la familia Añaños), instalada en Tailandia.

Con datos del período 1998-2002 se puede observar que China viene siendo el principal destino de las exportaciones peruanas al continente asiático, seguido de Japón. El cuadro muestra los montos de exportaciones en miles de dólares, y como se verá más adelante, muestra una clara tendencia ascendente en algunos países.

Para analizar el comportamiento de las exportaciones peruanas al continente asiático presentamos el Gráfico 2, donde se puede observar la tendencia claramente creciente de las exportaciones con destino a China, tendencia que comienza a finales de los 90, con un breve periodo de estancamiento para después volver a crecer. Es así que en 1998 las exportaciones peruanas a China ascendían a 233 millones de dólares incrementándose a 596 millones de dólares en el 2002 (más del 100\%). El otro socio comercial asiático importante es Japón, cuyas exportaciones con destino a Perú subieron de 216 millones de dólares en 1998, a 372 millones de dólares en el 2002. En el Gráfico 2 también se puede observar la tendencia de las exportaciones al Japón, que presentó un rápido crecimiento en los primeros años de la presente década, aunque luego disminuyó, tendencia que se mantiene a una menor tasa.

Si bien es cierto que el Perú siempre ha mantenido relaciones cordiales con Asia, éstas no han sido intensas y no son producto de una política de integración bien definida. En la década de los noventa comenzaron a establecerse relaciones más fluidas. En ésa época el Perú solo mantenía embajadas en China, Japón y las dos Coreas y es a raíz del creciente dinamismo económico de la región -el milagro económico de los tigres asiáticos-, el impresionante crecimiento de Tailandia y Malasia, y en menor medida el desarrollo de Filipinas e Indonesia, que el Perú comienza a estrechar sus lazos con Asia.

En primer lugar el gobierno peruano establece embajadas en Tailandia, Singapur, Malasia, Indonesia entre otros, a fin de mejorar los lazos políticos y comerciales con dichos países; luego se gestionó su admisión en el Foro de Cooperación Económica del Asia Pacífico (APEC), logrando el acceso en 1998, en la cumbre de líderes del foro llevado a cabo en Kuala Lumpur, Malasia.

El acceso del Perú al APEC ha permitido darnos a conocer a todos los países del Asia, que pudieron conocer las reformas que veníamos llevando a cabo en dicha época, haciendo del Perú un país con características atractivas en términos de modernización de los procedimientos de comercio exterior, defensa de los derechos de propiedad y del consumidor, así como la protección de la competencia como mejor mecanismo de asignación de recursos.

Como siguiente paso en el camino de acercamiento con los países del Asia, el Perú inicia el proceso de fortalecimiento de las relaciones comerciales con el este de Asia, que estaban básicamente orientadas al establecimiento de acuerdos de libre comercio a través de procesos de negociación. Es por ello que actualmente se vienen negociando tratados con Tailandia y Singapur. Si bien es cierto que el mundo viene experimentando un proceso de integración, el establecimiento de relaciones comerciales con Asia se hace más viable gracias a la participación del Perú en el APEC.

Es en este sentido que el Perú ha establecido otro tipo de acuerdos con los demás países de Asia: se trata de acuerdos bilaterales para promover la inversión en nuestro país. A la fecha tenemos acuerdos de este tipo con Malasia, Corea, China, Singapur y Tailandia.

En resumen, la meta es hacer del Perú un hub (centro) de servicios, de manera que todo el flujo comercial entre Asia y América del Sur pase por nuestro país. Para lograr esto se vienen implementando proyectos de integración basados en proyectos de infraestructura vial. El gobierno peruano viene participando activamente en la Iniciativa de Integración de la Infraestructura Regional Sudamericana (IIRSA), la que pretende conectar de manera multimodal a América del Sur, con el objetivo de implementar zonas de influencia. Los países miembros ya han 
acordado cuáles serán las rutas, y el Perú contará con tres ejes: el eje norte que conectará Piura con Manaos; el eje sur lo hará entre Ilo Matarani con el estado brasileño de Acre; y el eje centro unirá el puerto del Callao con Brasil, vía Pucallpa.

La ubicación geográfica del Perú es una gran ventaja con miras al logro de los objetivos trazados, ya que nos ubicamos en la parte media del lado occidental del Pacífico Sur, lo que brinda una clara ventaja para ser elegido como centro, de modo que podamos brindar servicios portuarios y aeroportuarios al comercio entre América del Sur y Asia, poniéndonos en ventaja frente a otros países de la región para desarrollar y ofrecer servicios conexos a la actividad como son los servicios financieros, seguros, logística y almacenaje, de procesamiento primario, transporte, comunicaciones y telecomunicaciones, entre otros.

Por lo descrito, el Perú en el año 2008 tendrá el gran desafío de consolidarse como país centro desde la perspectiva de Latino América del APEC, por lo que es necesario seguir impulsando acuerdos comerciales con Asia, en especial con China, Japón, Corea e India; así como seguir fortaleciendo reglas claras parar atraer las inversiones, derechos de propiedad y condiciones estables.

\section{RESPECTO AL TLC}

\section{¿Que es el TLC?}

Es un Tratado de Libre Comercio que permite un acuerdo entre dos o más países, en el que se establece no solo la eliminación progresiva de los aranceles y barreras para arancelarias, sino que va más allá de eso, tratando diferentes áreas como la inversión, derechos de propiedad intelectual, políticas de competencia, legislación laboral y ambiental, entre otros. No se encuentra dentro de las etapas de integración económica, debido a que es más que una zona de libre comercio pero no constituye necesariamente una unión aduanera. De este modo se puede considerar como un proceso paralelo, con un objetivo en común: la apertura comercial.

Si tomamos la acepción mas académica de un TLC, podemos coincidir con lo mencionado en el párrafo anterior; es decir, un TLC es un acuer- do comercial que busca la eliminación de las restricciones al comercio internacional.

Las restricciones al comercio internacional son medidas que establecen los países con la finalidad de limitar el acceso de productos extranjeros a sus mercados. Esta limitación no siempre genera beneficios para la sociedad que lo aplica; sin embargo, es una herramienta de política comercial con la que cuentan los gobiernos, y que en determinados momentos pueden usar para proteger ciertos sectores sensibles de su economía, mediante compensaciones y reconversiones del sector atrasado, aplicando tecnología e inversión.

Todo TLC es firmado por dos o más países, y normalmente se aplica sobre todas las partidas arancelarias. Pero además de ello, involucra la firma de acuerdos sobre determinadas legislaciones nacionales, en campos como el laboral, donde las negociaciones llevan a establecer las condiciones laborales que deben respetar los países firmantes, con la finalidad de crear condiciones de trabajo conforme a las normas internacionales.

En estos acuerdos también se incorporan negociaciones sobre propiedad intelectual, legislación ambiental y otros, áreas que están siendo discutidas dentro del marco de la Organización Mundial del Comercio (OMC), sin embargo, el paso lento que tienen estas discusiones "multilaterales" hace que los TLCs sean atajos para llegar más rápido a estos objetivos.

Una vez que sabemos que los TLCs son Acuerdos Comerciales, debemos de mencionar que el comercio involucra diferentes componentes que es necesario incorporar en una negociación de índole comercial, con la finalidad de cubrir todos los aspectos relevantes para la toma de decisiones de negocios. Es así que los acuerdos sobre las distintas legislaciones buscan crear condiciones similares para las economías que tendrán un libre comercio; es decir, que no existan países que tengan condiciones mas favorables que otros, y que hagan desleal la competencia entre los productores de los países firmantes.

Respecto a bloques y acuerdos comerciales existentes en este momento, en el mundo se desarrollan decenas de acuerdos comerciales, los mismos que involucran países de diferentes regiones, diferentes grados de desarrollo, de cul- 
turas y legislación. Los acuerdos de integración regionales o bilaterales son atajos, como mencionamos anteriormente, para los acuerdos multilaterales más amplios, los mismos que son el objetivo ultimo de la integración mundial. Sin embargo siempre es bueno observar cómo evolucionan los distintos acuerdos regionales o bilaterales para esbozar un escenario propicio para nuestro país, que está inmerso en diferentes frentes comerciales.

Los ejemplos más cercanos a la realidad peruana son los que involucran acuerdos de integración económica regional, especialmente de países latinoamericanos, que son los más cercanos a nuestra realidad.

Sobre ello podemos decir que entre los países latinoamericanos existen distintos estadíos de evolución, con diferencias económicas entre los países más grandes (Argentina, Brasil y México), algunas economías que han evolucionado de manera interesante durante las últimas décadas (Chile y Costa Rica), y los demás países que comparten indicadores socio-económicos similares.

Ahora describiremos los diferentes bloques comerciales que existen en América Latina.

\section{Comunidad Andina de Naciones (CAN)}

Es el bloque de integración vigente más antiguo de Sudamérica. Es un bloque comercial que se origina en 1969 en Colombia, con la suscripción del Acuerdo de Cartagena. Los miembros fundadores de este acuerdo regional (llamado en aquel entonces Pacto Andino), fueron Colombia, Bolivia, Chile, Ecuador y Perú. En 1973 se incorpora Venezuela y en 1976 se retira Chile.
El proyecto andino nació como respuesta frente a la insatisfacción de sus fundadores, ante la velocidad de avance de la Asociación Latinoamericana de Libre Comercio (ALALC) y al tratamiento dado en éste foro a los países de menor desarrollo relativo. Fue concebido como un medio para adelantar el proceso de integración regional. El Acuerdo planteó, desde sus inicios, un proceso de integración más profundo que el de una zona de libre comercio o una Unión Aduanera. Contenía mecanismos que se enmarcaban dentro de la política de sustitución de importaciones imperante en la región para promover la industrialización y el comercio en los países andinos.

En la actualidad, la Comunidad Andina se encuentra en una fase de relanzamiento (últimos diez años), después de haber estado en un letargo de más de dos décadas. Es así, que los indicadores económicos de los últimos tiempos muestran una clara tendencia de incremento de los flujos comerciales dentro de la región; sin embargo, ésta no termina de ser el mercado más importante para la producción de sus países miembros, a pesar de ser los mercados más cercanos. Esto se debe principalmente al hecho que los países andinos más que complementarios, son competidores, lo que hace que sus mercados naturales no sean sus vecinos sino territorios más allá de sus fronteras regionales.

Como se observa en el Cuadro 3, la participación de las exportaciones de cada país a la CAN, en comparación con el resto del mundo, indica el grado de comercio existente entre los países miembros. Las estadísticas mostradas van desde 1995 hasta el 2003, y en ellas se ve que la tendencia en el último decenio ha sido estable y ha girado alrededor del $10 \%$ de las expor-

Cuadro 3

Participación anual de las exportaciones intrarregionales comparadas con el mundo

\begin{tabular}{|c|c|c|c|c|c|c|}
\hline Año & CAN & Bolivia & Colombia & Ecuador & Perú & Venezuela \\
\hline 1995 & 12,7 & 19,2 & 19,8 & 8,2 & 7,4 & 10,9 \\
\hline 1996 & 10,3 & 24,1 & 17,4 & 8,7 & 7,2 & 7,6 \\
\hline 1997 & 11,8 & 19,7 & 18,4 & 12,1 & 7,6 & 9,2 \\
\hline 1998 & 13,9 & 24,1 & 19,7 & 13,0 & 8,3 & 11,5 \\
\hline 1999 & 9,1 & 20,9 & 14,1 & 10,6 & 5,8 & 6,1 \\
\hline 2000 & 9,0 & 21,3 & 16,6 & 13,7 & 6,6 & 5,1 \\
\hline 2001 & 11,1 & 27,2 & 22,3 & 17,2 & 7,4 & 4,9 \\
\hline 2002 & 10,8 & 28,6 & 19,5 & 16,0 & 6,6 & 5,4 \\
\hline 2003 & 8,9 & 25,8 & 14,2 & 17,2 & 6,3 & 4,2 \\
\hline
\end{tabular}


taciones. Para algunos países como Colombia, Ecuador y Bolivia, el mercado regional ha sido importante; a la vez que para el Perú y Venezuela ha sido un mercado interesante, pero no el más importante.

\section{MERCOSUR}

Otro acuerdo comercial importante vigente en Latino América es MERCOSUR, bloque conformado por Argentina, Paraguay, Uruguay y Brasil que surgió en la década de los noventa como un proceso acelerado de integración de las economías de la parte oriental de América. Así, el objetivo primordial del MERCOSUR es la integración de los cuatro Estados involucrados, a través de la libre circulación de bienes, servicios, factores productivos, establecimiento de un arancel externo común, adopción de una política comercial común, coordinación de políticas macroeconómicas y sectoriales, y la armonización de legislaciones en las áreas pertinentes, todo ello orientado a lograr el fortalecimiento del proceso de integración, donde la zona de libre comercio y la unión aduanera constituyen pasos intermedios para alcanzar un mercado único que genere un mayor crecimiento de sus economías, aprovechando el efecto multiplicador de la especialización, las economías de escala y el mayor poder negociador del bloque.

Cabe destacar, que de acuerdo a las estadísticas del 2005 de la Comisión de Promoción de las Exportaciones (PROMPEX) (ver www.prompex.gob.pe), MERCOSUR es un mercado para las exportaciones peruanas cercano a los 510 millones de dólares. Es decir, cerca del 3\% de las exportaciones comerciales del Perú se van a los países del bloque MERCOSUR. A nivel global, no es uno de los socio comerciales mas importante, sin embargo cabe destacar que el Perú no tiene un acuerdo de libre comercio con estos países, lo cual puede explicar de alguna manera el bajo comercio existente.

\section{Acuerdo de Libre Comercio de América del Norte (NAFTA)}

Un último acuerdo que queremos revisar a manera de ejemplo de acuerdos regionales de libre comercio, es el NAFTA (Acuerdo de Libre Comercio de América del Norte, por sus siglas en ingles), establecido en 1994. Este acuerdo involucra a tres países del Norte de América, como son Canadá, Estados Unidos y México. Es un tratado comercial que prevé la eliminación de los derechos aduaneros en los intercambios de sus países miembros. Asimismo ha liberalizado sustancialmente el comercio de servicios y ha abierto los mercados públicos, incluyendo sectores de servicios y construcción. El acuerdo impide a las partes discriminar entre productores nacionales y productores extranjeros en el comercio de servicios, mercado público e inversiones. Todos los servicios están incluidos dentro del acuerdo, siempre y cuando no hayan sido exentos explícitamente de las disposiciones del NAFTA. El acuerdo enumera en un anexo las exclusiones referidas por los diferentes niveles de gobierno dentro de cada país.

Lo interesante del NAFTA es que es el primer acuerdo de integración comercial que incorpora a países con grados de desarrollo distintos; es así que un país como México, con indicadores de desarrollo menores que sus socios comerciales, se embarcó con ellos en una región comercial que ha generado economías positivas para los tres miembros del acuerdo. Este TLC ha sido el ejemplo para los demás países que han querido firmar acuerdos de libre comercio con Estados Unidos, un país que por sí solo representa una cuarta parte del consumo mundial.

Para demostrar que este tipo de acuerdos genera efectos positivos para los países que lo suscriben, especialmente para aquellos que tienen menor grado de desarrollo, veremos algunas estadísticas que dan luces sobre el asunto, que no deben sesgarnos porque siempre es necesario tener una mirada crítica a estos acuerdos con la finalidad de lograr los mayores beneficios para nuestros países.

Como se aprecia en el gráfico de la siguiente página, las exportaciones mexicanas han crecido sustancialmente ha partir de la fecha de suscripción del NAFTA, además de la creación de 8.2 millones de nuevos empleos. Otro dato interesante es que uno de cada cinco nuevos empleos creados en México está relacionado con el incremento de la exportación, con un ingreso superior en $40 \%$ en promedio respecto de otros empleos. Asimismo las inversiones extranjeras en México se incrementaron en $40 \%$ y las exportaciones mexicanas a Estados Unidos crecieron más del 166\% entre 1994 y $2004^{1}$. 
Gráfico 3

Evolución de las exportaciones mexicanas

(millones de dólares)

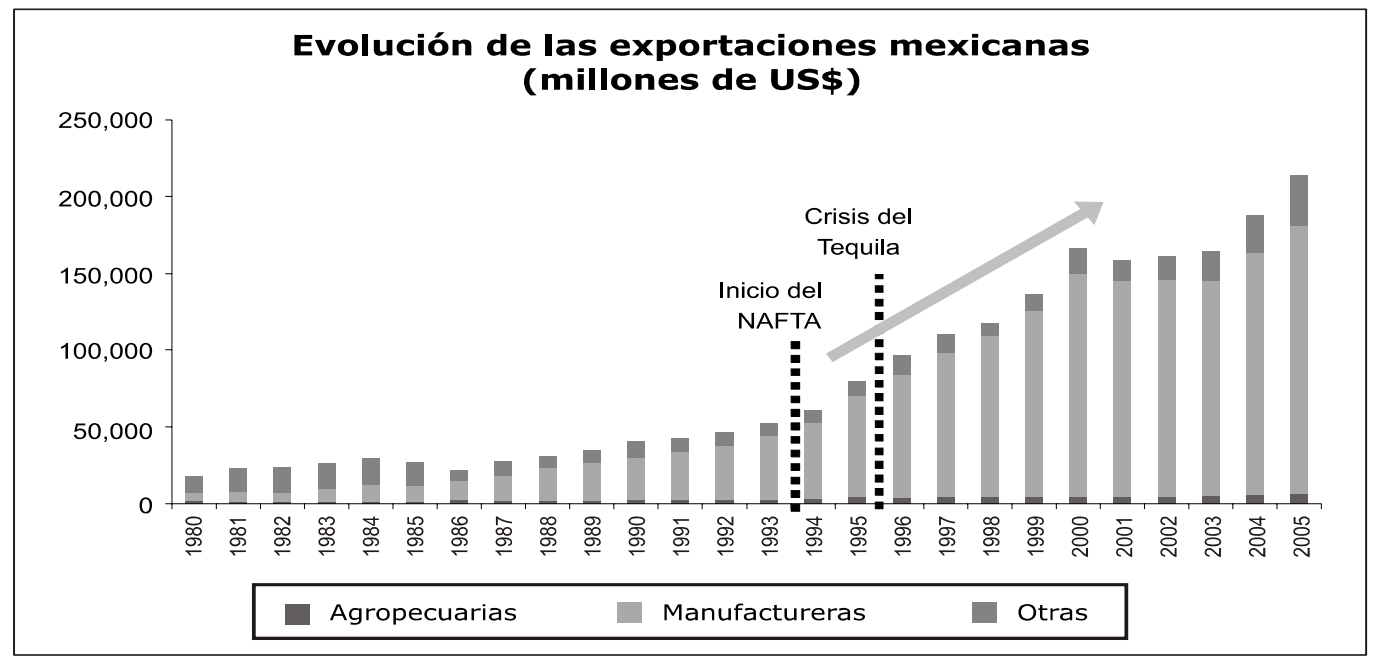

Fuente: Banco de México, INEGI, Secretaría de Economía de México. Elaboración: COMEXPERU.

\section{Cuadro 4}

Exportaciones del Perú al Mundo y a países después de firmar Acuerdos Preferenciales Parciales con los países mencionados

\begin{tabular}{|c|c|c|c|c|}
\hline Año & Valor Tot Mundo & Valor Chile & Valor Mexico & Valor USA \\
\hline 1998 & $5,725,836,035.70$ & $138,649,463.24$ & $137,287,258.94$ & $1,856,919,481.93$ \\
1999 & $6,043,505,004.45$ & $172,689,574.28$ & $171,091,469.75$ & $1,770,183,732.60$ \\
2000 & $6,882,787,213.78$ & $264,789,482.04$ & $150,532,205.97$ & $1,905,410,079.82$ \\
2001 & $6,956,036,877.16$ & $282,759,577.75$ & $128,077,105.11$ & $1,749,702,799.01$ \\
2002 & $7,665,140,320.25$ & $254,539,035.96$ & $129,739,357.74$ & $1,979,730,140.56$ \\
2003 & $9,026,650,694.50$ & $412,576,551.69$ & $108,077,358.33$ & $2,409,813,603.19$ \\
2004 & $12,726,497,518.78$ & $719,064,502.89$ & $229,057,258.41$ & $3,708,187,628.04$ \\
2005 & $17,172,819,014.77$ & $1,129,406,622.33$ & $332,060,795.82$ & $5,269,188,104.97$ \\
\hline
\end{tabular}

* Acuerdo Firmado en 1998

** Inicio de Negociaciones en 1996

Fuente: PROMPEX www.prompex.gob.pe

*** ATPDEA Firmado en 1991 y vence en 2006

Por otro lado, si vemos el Cuadro 4 encontraremos algunas estadísticas sobre el flujo comercial del Perú con países con los cuales tenemos Acuerdos Preferenciales Parciales (APP), como son México y Chile dentro del marco de ALADI, y con Estados Unidos dentro del marco de la ATPDEA; podemos ver que los flujos se han incrementado sustancialmente en los últimos años, debido principalmente al acceso preferencial a estos mercados. En el caso de México y Chile estos acuerdos son recíprocos y permiten tanto a ellos como a nosotros intercambiar bienes con aranceles preferenciales, que han tenido un efecto bastante positivo como mercado destino de las exportaciones peruanas. Así vemos que el mercado chileno, país con el que firmamos un APP en 1998, ha crecido desde un monto cercano a los 140 millones de dólares en el año de suscripción del acuerdo, hasta la importante cifra de 1,130 millones de dólares en el 2005, convirtiéndolo en nuestro tercer mercado destino.

Para el caso de México, hemos iniciado las negociaciones en 1996 y alcanzado mayores avances a partir de 1998; esto ha hecho que nuestras exportaciones a México se hayan duplicado en menos de diez años. Los datos referidos a Estados Unidos los analizaremos cuando veamos el ATPDEA.

Todos estos argumentos estadísticos nos dan referencia sobre las oportunidades que el Perú podría obtener en el caso de aprobarse un acuerdo de libre comercio con nuestro socio co- 
mercial del norte. Ya vimos con los datos precedentes, que nuestros acuerdos de preferencias parciales con algunos países nos han abierto mercados importantes; sin embargo, algunos acuerdos como la CAN y MERCOSUR no han significado importantes aportes debido a diversos factores, siendo el de mayor relevancia el de no contar con acuerdos comerciales de libre comercio de bienes y servicios. Sobre lo último cabe mencionar que hay avances dentro del marco de la CAN, pero que aún no se traducen en cifras.

\section{Ley de Promoción Comercial Andina y Erradicación de la Droga (ATPDEA)}

La Ley de Preferencias Arancelarias Andinas ${ }^{2}$ (ATPA, por sus siglas en inglés), es un régimen de excepción otorgado unilateralmente por los Estados Unidos al Perú, Bolivia, Colombia y Ecuador para apoyar la lucha contra el tráfico ilícito de drogas. Fue otorgada por primera vez el 4 de diciembre de 1991, ofreciendo ingreso libre de aranceles a cerca de 5,500 productos, dónde no se encontraban incluidos textiles y confecciones, entre otros. Este primer acuerdo venció el 4 de diciembre del 2001. El objetivo era incentivar las exportaciones mediante el establecimiento de un mercado preferencial que generara fuentes de trabajo alternativas para apoyar la sustitución del cultivo de la hoja de coca, así como la reducción del narcotráfico.

La Ley de Promoción Comercial Andina y Erradicación de la Droga (ATPDEA) renovó hasta el 31 de diciembre del 2006 las preferencias arancelarias a los productos que se beneficiaban con el ATPA de 1991; es decir que desde el 7 de agosto del 2002 todos los productos que gozaban de los beneficios del ATPA continuaron con trato preferencial; adicionalmente desde el $1^{\text {o }}$ de octubre del 2002 y previa designación como país beneficiario por el Presidente de Estados Unidosse, se extienden dichos beneficios a:

- Nuevos productos como prendas de vestir elaboradas con insumos regionales, hasta por un monto equivalente al $2 \%$, en metros cuadrados, del total de prendas de vestir importadas por los Estados Unidos al mundo, incrementándose cada año hasta llegar al 5\% en el 2006. En el 2001 las exportaciones de prendas de vestir de los cuatro países andinos beneficiarios representaron, en metros cuadrados, menos del $0.88 \%$, lo que da la posibilidad al menos de duplicar las exportaciones de dichos productos en el primer año de vigencia del programa.

- Sin limitación de cuota a las prendas de vestir elaboradas con insumos de los Estados Unidos (maquila).

- Sin limitación de cuota a las prendas de vestir elaboradas con pelos finos de alpaca, llama y vicuña.

- Sin limitación de cuota al atún envasado al vacío en empaques flexibles ("tuna pouches", bolsas de aluminio, por ejemplo). No recibe beneficios el atún en conserva.

- Otros: calzado, petróleo o algún producto derivado del petróleo; relojes y partes de relojes $\mathrm{y}$, carteras y maletines de mano, guantes de trabajo y confecciones de cuero.

- El tratamiento preferencial arancelario NO se extiende a los siguientes productos: textiles (hilados y tejidos) y confecciones con excepción de las prendas de vestir (las prendas de vestir sí se benefician), ron y tafia (aguardiente de caña), azúcar, concentrados líquidos y productos que contengan azúcar (ejemplo: almíbar, jarabes) excedidos de la cuota fijada; atún preparado o preservado por cualquier medio en empaques al vacío (ejemplo: atún en conservas).

Como se puede observar de la información recogida del Ministerio de Comercio Exterior y Turismo, el ATPDEA ha sido un acuerdo que ha permitido al país (y los otros miembros Andinos) tener un acceso preferencial al mercado de Estados Unidos, esto de manera unilateral; es decir, ha habido una apertura temporal de las fronteras comerciales del país del norte más no de los países Andinos. Esta preferencia temporal ha tenido un impacto positivo para el caso del Perú.

Si revisamos las estadísticas mencionadas anteriormente en el Cuadro 4, podemos ver que las exportaciones del Perú se han ido incrementando a partir de la renovación del ATPDEA. Las estadísticas muestran que las exportaciones peruanas hacia los Estados Unidos se han casi cuadruplicado en los últimos siete años, siendo esto lo más significativo, ya que este mercado es históricamente el más importante para el Perú; representando casi el $30 \%$ de las exportaciones totales del Perú. 
Gráfico 4

Exportaciones del Perú al mundo y a países con ACE

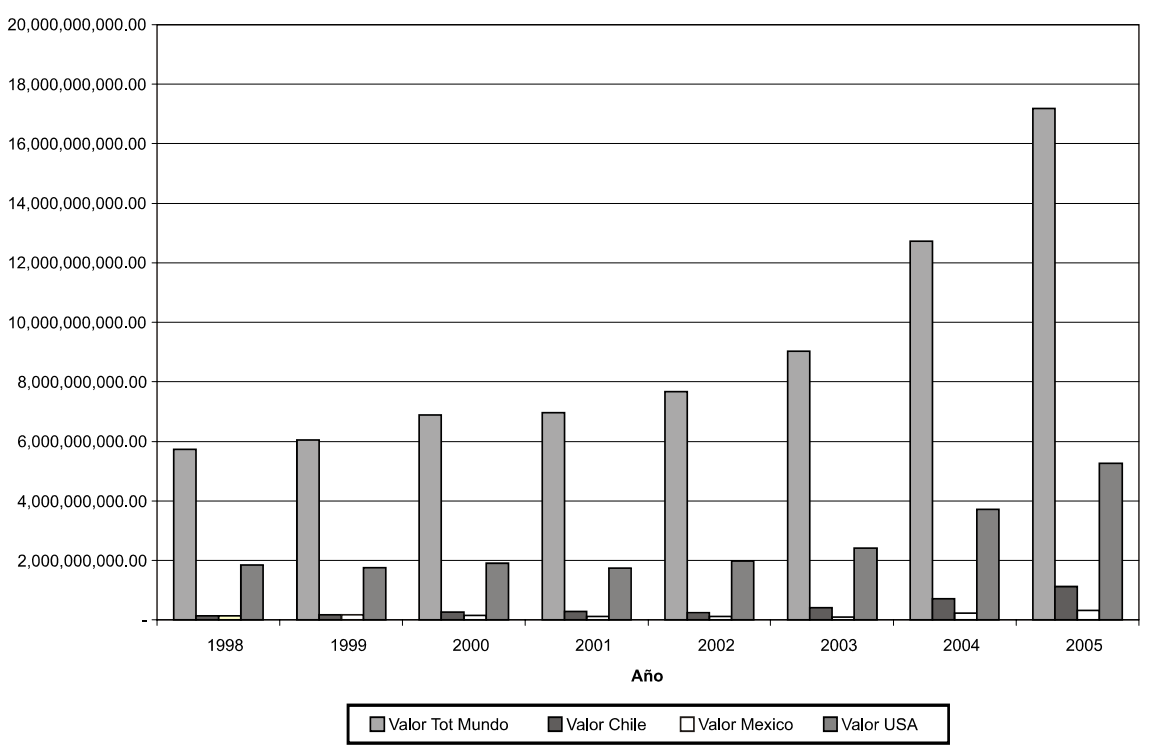

En el Gráfico 4 se puede apreciar la evolución de las exportaciones peruanas a los Estados Unidos en los últimos años, además de la evolución de las exportaciones peruanas al mundo y a dos de los países con los cuales tenemos acuerdos de acceso preferencial.

Como vemos en el gráfico, el Perú está viviendo un momento favorable en lo que se refiere a las exportaciones, especialmente en los últimos cinco años. Pero lo más destacable del grafico es apreciar como las exportaciones peruanas a los Estados Unidos crecen de manera sostenida en el mismo período, y esto se explica en el acceso preferencial que el Perú tiene dentro del marco del ATPDEA. Sin embargo, este acuerdo dejará de estar vigente a fines de diciembre del presente año; es decir, si no contamos con un acuerdo que permita prolongar en el tiempo el acceso preferencial a este mercado estaríamos enfrentando la posibilidad de que se frene el buen momento de la economía peruana, y con ello todo el sistema económico y social que está involucrado.

\section{SITUACION ACTUAL DEL TLC}

Luego de larga discusión de si llevar adelante el TLC con Estados Unidos o no, el Congreso de la República del Perú finalmente lo aprobó el 27 de Junio del 2006. Después de trece rondas de negociaciones realizadas a lo largo de dos años, el Perú y Estados Unidos firmaron el Tratado de Libre Comercio, acuerdo que para entrar en vigencia debe de ser ratificado por los congresos de ambos países, por lo que estaremos a la espera de la aprobación del Congreso de los Estados Unidos, cuya votación probablemente se realice a fines de año, con resultados todavía inciertos. Como la discusión está sobre la mesa, veremos como el TLC pueda estar afectando algunos sectores de la producción local y si éstos serán serán beneficiados o no con la entrada en vigencia del acuerdo; ello dependerá de los Programas de Compensaciones y del Programa de Reconversión a aplicar.

El sector Salud es uno de los temas más sensibles sobre todo para economías de bajos recursos como la nuestra, ya que existen posiciones encontradas. De acuerdo a la Asociación Nacional de Laboratorios Farmacéuticos, el TLC no tendrá efectos negativos sobre el precio de las medicinas, por lo que los medicamentos genéricos no serán retirados, tal como muchas voces han venido anunciando. Ello por cuanto el acuerdo no se aplica de manera retroactiva a los productos; es decir aquellos productos que ya existen en el mercado bajo esa forma de producción, continuarán existiendo. De otro lado, el libre comercio permitirá que los laboratorios norteamericanos, que cumplen con estándares bastante más elevados que los del mercado local, puedan vender sus productos en el país y competir en el segmento de los genéricos. Esto hará que los precios de los mismos bajen para el consumidor final. Asimismo, el precio de los medicamentos protegidos por patentes tendrían 


\section{Cuadro 5}

Comparación de algunos países con Estados Unidos

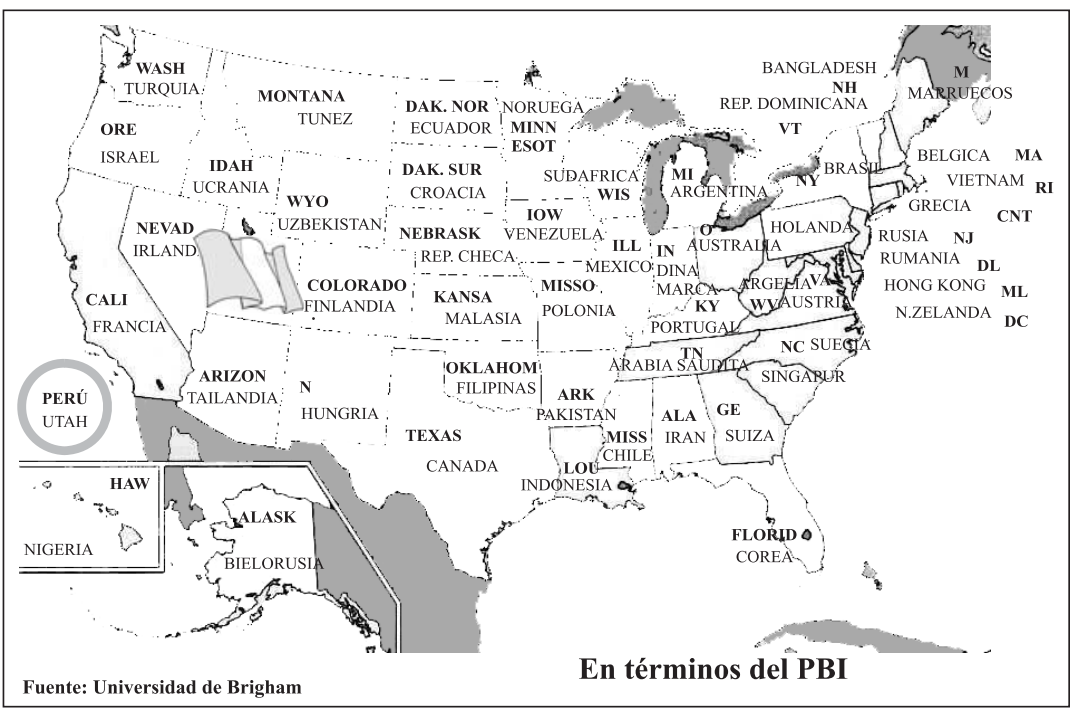

un periodo de protección más largo que podría implicar mayores gastos por parte de los pacientes que quieren acceder a los mismos. Sin embargo cabe mencionar que los medicamentos genéricos son alrededor del 95\% del total de medicamentos ${ }^{3}$, y seguirán siendo ofrecidos en el Sistema Nacional de Salud Publica.

En cuanto al sector agrario, hay tres productos que son especialmente sensibles para el Perú: trigo, maíz amarillo y algodón, para los cuales el gobierno está planteando medidas compensatorias que permitan a los agricultores de estos sectores tener ingresos suficientes que les permitan acceder a tecnología innovadora o, en el peor de los casos, reorientar la producción de sus terrenos hacia otros sectores más rentables de la economía. En el resto de productos agrícolas no se ha visto un efecto negativo sobre los mismos en el corto plazo. Es más, de acuerdo a un estudio elaborado por Centro de Estudios Económicos y Desarrollo Social (CEEDE) $)^{4}$ el TLC tendrá un efecto cercano a cero en la producción de papa de Apurímac. Es decir, existirían oportunidades para los productores de aquella región para que puedan exportar papa hacia Estados Unidos, en vez de verse afectados por el Tratado.

También en el sector agro-exportador podemos ver que el TLC tiene un efecto positivo. Las exportaciones no tradicionales peruanas, entre las que se encuentran el espárrago, alcachofa y otros productos agrícolas, se han incrementado notablemente gracias a los beneficios del acceso preferencial al mercado norteamericano, el cual se mantendría con la firma del TLC. Es así que estos sectores podrían incrementar sus posibilidades de venta, y por lo tanto, tendrían incentivos para incrementar la producción.

Ahora, si revisamos al sector textil, podemos ver que este sector ha tenido un boom en los últimos años gracias al ATPDEA, el cual podría prolongarse en el tiempo gracias al tratado. Este sector es especialmente importante para el Perú, por cuanto absorbe una gran masa de la PEA y es un sector dinamizador de la industria local. De acuerdo con algunos estudios elaborados por el MINCETUR ${ }^{5}$, el sector textil ha generado más de 800,000 puestos de trabajo en los últimos cinco años. Esta cifra da clara muestra de la relevancia de mantener los beneficios del acceso a este mercado.

El mercado de Estados Unidos resulta sumamente importante para nuestro país. Para darnos una idea clara de su potencial diremos que la producción total del Perú, en términos del PBI, equivale a la del estado de Utah, y en términos globales, el PBI del país del norte equivale a 180 veces el PBI del Perú.

\section{CONCLUSIONES}

Como podemos ver, el Perú está frente a una gran oportunidad, con un escenario extremadamente positivo en relación a su historia reciente por lo que es necesario llegar a la conclusión y aprobación de este acuerdo, pendiente de votación en el Congreso de Estados Unidos. 
Hemos visto cómo los diferentes sectores de la economía nacional se han beneficiado con el desarrollo del sector exportador; los ejemplos de nuestros acuerdos con otros países, aunados a los ejemplos de otros países con acuerdos similares al que queremos firmar, dan clara cuenta de que los efectos positivos del TLC son bastante más importantes que los negativos. En términos de costobeneficio resulta favorable para el país, ya que en la negociación uno de los involucrados no puede tener el $100 \%$ de los beneficios. Sin embargo, no hay que olvidar que la economía no se trata de cifras sino de personas, por lo que es necesario crear un sistema de protección a través de Programas de Compensaciones y de Reconversión para los sectores que se vean perjudicados por la firma del Tratado. Este sistema de protección no significa volver a un estado proteccionista y subsidiador de empresas o sectores ineficientes, sino todo lo contrario; es decir, debemos tener un estado promotor que aplique medidas económicas e instrumentos necesarios a estos sectores para ubicarlos en el nivel de competitividad de los productores de Estados Unidos.

Para el caso de aquellos sectores donde el desnivel sea muy profundo, el Estado debe jugar un rol orientador de la producción con la finalidad de que quienes trabajan en esos sectores puedan reorientar su producción hacia otros más lucrativos o rentables, mediante programas de reconversión que permitan incluirlos en el sistema y el mercado. Cabe advertir que si el TLC produjera mayores perjuicios que beneficios se procedería a denunciar el tratado, dando por concluido el acuerdo.

De otro lado, debemos de recordar que el Perú es un país partido geográficamente, y que es necesario incorporar a las diferentes regiones dentro de este modelo económico orientado a las exportaciones, haciendo que éstas se vuelvan cadenas integradas de valor, que permitan a la población salir adelante y beneficiarse de manera conjunta de las mejoras económicas.

Finalmente, es necesario ver al Tratado de Libre Comercio no como una amenaza, sino más bien como una oportunidad, y seguir desarrollando más TLCs con la Unión Europea y otras economías. Consideramos que este tratado es una oportunidad en un mundo donde los que se queden rezagados en el camino del desarrollo, no podrán acceder al bienestar económico y seguirán siendo pobres.
Aprendamos de nuestra historia y en este contexto de globalización económica no cerremos nuestras fronteras, dejando de aprovechar una nueva oportunidad. No repitamos los errores de los años 50 y 60 de mirar hacia adentro cuando todo el mundo miraba hacia fuera. Estamos en un mundo globalizado, donde los mercados se integran y los pueblos que participan de esta globalización se ven beneficiados, mientras que los que se aíslan quedan rezagados en la historia y en el tiempo.

\section{NOTAS}

1 Fuente COMEXPERU, Yale Economic Review 2004, otros.

2 Mincetur: http://www.mincetur.gob.pe/COMERCIO/OTROS/ Atpdea/ley_atpa_atpdea/resumen_ejecutivo.htm.

3 Fuente ALAFARPE.

4 Centro de Estudios Económicos y Desarrollo Social. (2006). Documento de trabajo № 4, Lima.

5 MINCETUR: www.mincetur.gob.pe.

\section{BIBLIOGRAFIA}

Andriamananjara, Soamiely y Schiff, Maurice. (1998). Regional groupings among microstates. World Bank.

Chan, Julio. Avances y perspectivas en las relaciones Perú - Asia. Portal de la Pontificia Universidad Católica del Perú (PUCP).

Comisión de Promoción de las Exportaciones (PROMPEX). Ver en Página web: www.prompex.gob.pe

Comunidad Andina (2004). 35 years of commercial and economic integration in the Andean Community. Perú, General Secretary.

Comunidad Andina. (2004). 35 years of commercial integration. Lima, General Secretary, Statistic Document.

DG Trade (2006). EU: bilateral trade and trade with the world. Statistics.

Fondo Monetario Internacional. (2006). Nuevos Aires en Asia. En Revista Finanzas y Desarrollo (Junio 2006).Ministerio de Comercio Exterior y Turismo (MINCETUR). Ver Página web: www.mincetur.gob.pe.

Instituto Nacional de Estadística e Informática (INEI). Página web: www.inei.gob.pe

Romalis, John. Nafta's and Cusfta's Impact On International Trade. Cambridge, National Bureau Of Economic Research. 